\title{
Adenovirus-mediated Drosophila melanogaster deoxyribonucleoside kinase mutants combined with gemcitabine harbor a safe cancer treatment profile
}

\author{
ZHI ZHU ${ }^{1}$, SHUAI MA ${ }^{1}$, LEI ZHAO ${ }^{3}$, ZHE SUN $^{1}$, ANNING HE $^{2}$, HUIMIAN XU $^{1}$ and XINYU ZHENG ${ }^{1,2}$ \\ ${ }^{1}$ Department of Surgical Oncology, Department of General Surgery, First Affiliated Hospital, ${ }^{2}$ Lab. 1, Cancer Institute, \\ ${ }^{3}$ Center of Experiment Technology and Medical Research, China Medical University, Shenyang, P.R. China
}

Received August 22, 2010; Accepted October 13, 2010

DOI: $10.3892 /$ ijo.2010.887

\begin{abstract}
The purpose of this analysis was to investigate the enzyme activity and specificity of adenovirus-mediated Drosophila melanogaster deoxyribonucleoside kinase (Dm$\mathrm{dNK}$ ) mutants in combination with gemcitabine. Compared to herpes simplex type 1 thymidine kinases (HSV-TK) and other known dNKs, this Dm-dNK enzyme has a broader substrate specificity and a higher catalytic rate. We created the Dm-dNK mutants (dNKmut) by site-directed mutagenesis at the sites of $244 \mathrm{E}, 245 \mathrm{~S}, 251 \mathrm{~S}$, and $252 \mathrm{R}$, with the last 10 amino acids in the amino acid sequence randomly alternated. We subsequently evaluated the enzyme activity and substrate specificity. The engineered enzymes showed a relative increase in phosphorylation in the nucleoside analogs of gemcitabine ( $\mathrm{dFdC}, 2^{\prime}, 2^{\prime}$-difluoro-deoxycytidine) compared to the wild-type enzyme. The dNKmut enzymes were expressed in breast (Bcap37) and gastric (SGC-7901) cancer cell lines. In studying the sensitivity of the cell lines to $\mathrm{dFdC}$, conditionally replicative adenovirus (CRAd) ZD55-dNKmut showed higher expression and enzymatic activity than the replication-defective adenovirus Ad-dNKmut in cancer cells, but with less cytotoxicity to cancer cells than that of Ad-dNKmut. Our data suggest that the triple phosphorylated $\mathrm{dFdC}$ catalyzed by dNKmut inhibited the replication of adenovirus with a simultaneous positive therapeutic effect on cancer cells. Therefore, concomitant use of the ZD55-dNKmut and dFdC could be a novel targeted strategy in suicide gene therapy with safe control of excessive virus replication.
\end{abstract}

Correspondence to: Dr Xinyu Zheng, Department of Surgical Oncology, Department of General Surgery, First Affiliated Hospital, China Medical University, Shenyang 11001, P.R. China

E-mail: xyzheng@mail.cmu.edu.cn

Key words: Drosophila melanogaster multisubstrate deoxyribonucleoside kinase, oncolytic adenovirus, virus therapy, suicide gene therapy, safe control

\section{Introduction}

Nucleoside kinases have been studied for possible use in suicide gene applications. We were interested in studying the multisubstrate deoxyribonucleoside kinase of Drosophila melanogaster (Dm-dNK), which phosphorylates purine and pyrimidine deoxyribonucleosides as well as several anti-viral and anti-cancerous nucleoside analogs (1). The broad substrate specificity of this enzyme, along with its high catalytic rate, makes it unique among the known dNKs. Expression of $\mathrm{Dm}-\mathrm{dNK}$ in cancer cells increases the sensitivity to several cytotoxic nucleoside analogs (2-4), rendering the enzyme a candidate for possible use as a suicide gene with combined gene therapy and chemotherapy.

Aligned with other deoxyribonucleoside kinases, homologous amino acid patches are distributed over the entire amino acid sequence of Dm-dNK, except for the C-terminal part. The last 10 amino acids of the very $\mathrm{C}$-terminal part are unique and not only influence catalytic efficiency for thymidine but also involve nuclear localization signals. The role of the C-terminal part of the enzyme was investigated in detail by construction and expression of a 10 amino acid deletion mutant. The $\Delta 10 \mathrm{Dm}-\mathrm{dNK}$ mutant has an even higher catalytic rate for deoxyribonucleosides compared with the wild-type enzyme (5). To further explore the phosphorylation capacity of mutagenesis, we designed and constructed mutated Dm-dNK (Dm-dNKmut) with the last 10 amino acids randomly alternating at the sites of $244 \mathrm{E}, 245 \mathrm{~S}, 251 \mathrm{~S}$, and $252 \mathrm{R}$ in the amino acid sequence. We wished to study its enzymatic properties in a functional assay after cellular expression.

We explored the pyrimidine gemcitabine $(\mathrm{dFdC})$, which is an anti-tumor agent and a more efficient and selective substrate for Dm-dNK and its mutants (6-8). $\mathrm{dFdC}$ is known to enhance cell killing activity by phosphorylation of enzymes. However, our use of mutants combined with $\mathrm{dFdC}$ has as-yet to be studied in suicide gene therapy or in an in vivo animal model. Therefore, we constructed the $\mathrm{dNKmut} / \mathrm{dFdC}$ system to evaluate the efficacy of our approach in the prevention of breast and gastric cancer. It is well known that during suicide gene therapy, nucleoside analogs are phosphorylated by suicide enzymes and then further phosphorylated by cellular enzymes. 
These compounds ultimately convert to their triphosphate forms (TP), which, upon incorporation into the DNA of tumor cells, result in blocking DNA synthesis through chain termination, activation of the DNA damage response, induction of cell death, and inhibition of cell proliferation $(9,10)$. $\mathrm{dFdC-TP,}$ which is generated by the new system, harbors a putative killing effect to the adenovirus (Ad) and may lead to further apoptosis of cancer cells and blocking of the superfluous replication of the adenovirus. This hypothesis may explain why studies that have demonstrated anti-tumor efficacy of HSV-TK expressing oncolytic adenoviruses could not be augmented by ganciclovir (GCV) treatment (11-13). The contrary conclusion may be due to the inhibited expression of HSV-TK by GCV-TP through inducing the death of viruses.

In this study, we take advantage of the conditionally replicative adenovirus (CRAd) ZD55 vector (14) and the replication-deficient adenovirus (RDAd) Ad vector to construct ZD55-dNKmut and Ad-dNKmut, respectively. Previous studies consistently demonstrate that CRAd carrying a therapeutic gene mediated stronger anti-tumor efficacy than RDAd (15-18). Severe adenoviral deluge is the key obstacle of CRAd in the treatment of cancer, including gene therapy and chemotherapy (19). However, these scenarios did not occur with our approach. Instead, we found the combinations of suboptimal doses of ZD55-dNKmut with dFdC targeting breast and gastric cancer cells to potently block the excessive replication of adenovirus and protect the normal cells. The oncolytic effect or cytotoxicity of CRAd is not obvious in the application of suicide gene therapy when compared with ZD55-dNKmut and Ad-dNKmut, despite the fact that a higher expression of enzyme was obtained by ZD55-dNKmut. Therefore, ZD55-dNKmut/dFdC could be a novel targeted strategy in suicide gene therapy with safe control of excessive virus replication.

\section{Materials and methods}

Cells and cell cultures. The human breast cancer cell line, Bcap37, and the human gastric cancer cell line, SGC-7901, were both purchased from the Institute of Cell Biology, Chinese Academy of Sciences (Shanghai, China). MRC-5, the human embryo lung fibroblast cell line, and HEK293, the human embryonic kidney cell line, were both purchased from American Type Culture Collection (Manassas, VA, USA). SGC-7901 and HEK293 were cultured in Dulbecco's modified Eagle's medium (DMEM, Gibco, Grand Island, NY) supplemented with $10 \%$ heat-inactivated fetal bovine serum (FBS, Gibco) and incubated at $37^{\circ} \mathrm{C}, 5 \% \mathrm{CO}_{2}$. Bcap37 and MRC-5 were cultured in MEM (Gibco) or RPMI-1640 (Gibco) supplemented with $10 \%$ heat-inactivated FBS and incubated at $37^{\circ} \mathrm{C}, 5 \% \mathrm{CO}_{2}$.

Construction of recombinant adenovirus. The complete cDNA sequence of Dm-dNKmut gene was released from plasmid pGEM-T-dNKmut with endonucleases EcoRI and BamHI (New England Biolabs, Beverley, MA) and ligated into plasmid pENTR13 [made by ourselves, which contains the sequence of mouse cytomegalovirus $(\mathrm{CMV})$ promoter $+\operatorname{His}-\operatorname{tag}(\mathrm{N})+$ multiple clone site + SV40 polyA] to generate pENTR13-
dNKmut. pENTR13-dNKmut was digested with endonucleases AgeI and NotI (New England Biolabs), a 1317-bp fragment expression cassette of dNKmut containing mouse CMV promoter + His-tag $(\mathrm{N})+$ dNKmut gene + SV40 polyA was excised and inserted into the AgeI and NotI sites of pZD55 to generate pZD55-dNKmut, and plasmid pShuttle-basic (Sinogenomax, Inc., Beijing, China) to generate shuttle plasmid pShuttle-basic-His-tag(N)-dNKmut (Ad-dNKmut). Plasmid pZD55-dNKmut and Ad-dNKmut were individually transfected into HEK293 cells using Lipofectamine 2000 (Life Technologies) combined with adenovirus packaging plasmid pBGHlox (Microbix Biosystems). After homologous recombination, we obtained a replication-deficient adenovirus known as Ad-dNKmut, and a replicative adenovirus known as ZD55-dNKmut, in which the viral E1B55-kDa gene was deleted. Large-scale amplification of adenoviruses was carried out in HEK293 cells and purified by ultracentrifugation with cesium chloride.

RT-PCR analysis. Bcap37, SGC-7901, and MRC-5 cells were infected with Ad-dNKmut or ZD55-dNKmut at a multiplicity of infection (MOI) rate of 10. Two days after infection, cells were purified with a TRIzol (Sigma, USA) method and then assayed for Dm-dNKmut gene expression using the RT-polymerase chain reaction (PCR) kit (Takara, Japan). This manipulation was done according to the manufacturer's instructions. A cDNA equivalent of $1 \mathrm{ng}$ of RNA was amplified by PCR using primers specific for the target genes. The thermal cycles were: $94^{\circ} \mathrm{C}$ for $1 \mathrm{~min}, 55^{\circ} \mathrm{C}$ for $1 \mathrm{~min}, 72^{\circ} \mathrm{C}$ for $1.5 \mathrm{~min}$ for 35 cycles for dNKmut (785 bp); and $94^{\circ} \mathrm{C}$ for $1 \mathrm{~min}, 55^{\circ} \mathrm{C}$ for $1 \mathrm{~min}, 72^{\circ} \mathrm{C}$ for $1.5 \mathrm{~min}$ for 35 cycles for GAPDH (452 bp). Nucleotide sequences of dNKmut primers were as follows: sense 5'-AAGGACTGATGGCGGAGGCA-3'; antisense 5'-TTGTCGTACCTGGCGACCCTCTGGCT-3'. Nucleotide sequences of GAPDH primers were as follows: sense 5'-ACC ACAGTCCATGCCATC AC-3'; antisense 5'-TCCACCACC CTGTTGCTG TA-3'. The amplification products were separated by $2 \%$ agarose gel electrophoresis and visualized by SYBR Green staining.

Immunoblot analysis. Bcap37, SGC-7901, and MRC-5 cells were seeded in 24-well plates at a density of $5 \times 10^{4}$ cells/well, and infected with Ad-dNKmut or ZD55-dNKmut at a MOI rate of 1 . After 2 days, cells were rinsed 3 times with phosphatebuffered saline (PBS) and fixed in 4\% paraformaldehyde for 25 min. Immunoblotting was performed by incubation, first with mouse anti-human His-tag antibody (Merck, Germany) at $4^{\circ} \mathrm{C}$ for $1 \mathrm{~h}$, followed by incubation with goat anti-mouse IgG conjugated with TRITC (Santa Cruz Biotechnology, USA) at a dilution of 1:100. The labeled cells were observed under a fluorescent microscope (Olympus, Tokyo, Japan).

Enzyme assay. Bcap37, SGC-7901, and MRC-5 cells were seeded in 6-well plates at a density of $5 \times 10^{5}$ cells/well and cultured for $24 \mathrm{~h}$, followed by infection with ZD55-dNK and ZD55 at a MOI rate of 1 . Two days later, cell protein extracts were prepared as described (20). The assays were performed in $50 \mathrm{mM}$ of Tris- $\mathrm{HCl}$, a pH of 7.6, $5 \mathrm{mM}$ of $\mathrm{MgCl}_{2}, 5 \mathrm{mM}$ of ATP, $2 \mathrm{mM}$ of dithiothreitol, $15 \mathrm{mM}$ of NaF, $100 \mathrm{mM}$ of $\mathrm{KCl}, 0.5 \mathrm{mg} / \mathrm{ml}$ bovine serum albumin, and $0.6 \mathrm{mg}$ of protein 
extract in a total volume of $35 \mathrm{ml}$. We used $2.5 \mathrm{mM}$ of methyl${ }^{3} \mathrm{H}$-dThd (Moravek Biochem) in the assays and mixed with equivalent amounts of unlabeled substrates. Aliquots of the reaction mixture were spotted on Whatman DE-81 filters after 10-, 20-, and 30-min incubation at $37^{\circ} \mathrm{C}$. The filters were washed 3 times in $5 \mathrm{mM}$ of ammonium formate. The nucleoside monophosphates were eluted from the filter with $0.5 \mathrm{M}$ of $\mathrm{KCl}$, and the radioactivity was determined by scintillation counting.

Cytotoxicity assay. Cells were dispensed in 96-well plates (Corning, USA). The culture solution was removed on the second day and the following were added to each well: $100 \mu \mathrm{l}$ of serum-free DMEM and virus ZD55-dNKmut, ZD55, Ad-dNKmut, and Ad-blank at a MOI rate of 10. After 2 days of incubation, the nucleoside pro-drug $\mathrm{dFdC}$ was added at a concentration of $0,0.001,0.01,0.1,1$, and $10 \mu \mathrm{M}$ (mock was the drug-treated only group). After another 3 days, and a total of 5 days of incubation, cell viability was measured by the tetrazolium salt 3-(4,5-dimethylthiazol-2-yl)-2,5diphenyl tetrazolium bromide (MTT) assay (Promega, USA) to determine the combined cytotoxic effect. Absorbance was measured with an enzyme immunoassay instrument at $570 \mathrm{~nm}$, which serves as a measure of cell viability. Each data-point was generated from triplicate samples and repeated 3 times.

Flow cytometry apoptosis assays. For further clarification of the mechanism and effect of the combined suicide therapy, the apoptotic cells were detected by an Annexin V-FITC/PI double staining kit (Genmed Bioscience, China). Briefly, Bcap37 and MRC-5 cells were treated with $1 \mu \mathrm{M}$ of $\mathrm{dFdC}$ for 3 days after being infected with ZD55-dNKmut, ZD55, Ad-dNKmut, and Ad-blank for $48 \mathrm{~h}$, then trypsinized, pelleted, and washed in PBS. Cells were rinsed with a binding buffer and then resuspended with $200 \mu 1$ of the binding buffer. After adding $5 \mu \mathrm{l}$ of Annexin V-FITC $(20 \mu \mathrm{g} / \mathrm{ml})$ and $10 \mu \mathrm{l}$ of propidium iodide (PI, $50 \mu \mathrm{g} / \mathrm{ml}$ ), the mixture was incubated at room temperature for $10 \mathrm{~min}$ in the dark. The apoptosis ratio was analyzed using a FACScan flow cytometer [equipped with CellQuest and ModFITLT for Mac V1.01 software (Becton-Dickinson, San Jose, CA)].

Adenovirus replication assay. Bcap37, SGC-7901, and MRC-5 were cultured in 24-well dishes overnight and infected with ZD55-dNKmut, ZD55, and wild-type Ad at a MOI rate of 10 , then divided into two groups. One group was treated with $1 \mu \mathrm{M}$ of $\mathrm{dFdC}$ for 3 days. Two days after infection, a second group was only virus-treated for 5 days. Cells and media were then collected, respectively, freeze-thawed, and titered on HEK293 cells by the limiting dilution method (TCID50). Each assay was repeated 2-3 times, averaged, and expressed as $\mathrm{pfu} /$ cell $\pm \mathrm{SD}$.

Western blot analysis. Subconfluent cells were infected with viruses in the presence of $\mathrm{dFdC}$ as described above. Bcap37 and MRC- 5 cells were harvested 5 days post-infection and lysed in buffer ( $50 \mathrm{mmol} / \mathrm{l}$ Herpes, at a $\mathrm{pH}$ of $7.4,250 \mathrm{mmol} / \mathrm{l}$ $\mathrm{NaCl}, 1 \mathrm{mmol} / \mathrm{l}$ EDTA, $1 \mathrm{mmol} / \mathrm{l} \mathrm{DTT}, 1 \mathrm{mmol} / \mathrm{l} \mathrm{NaF}, 1 \%$ Triton X-100) containing protease inhibitors. Total protein (50-80 $\mu \mathrm{g}$ ) was separated on SDS-polyacrylamide gels under reducing conditions, transferred to polyvinylidene fluoride membranes (Millipore), and detected by the following antisera: rabbit anti-Ad2/Ad5 E1A at 1:1000 dilution (Santa Cruz Biotechnology) and goat anti- $\beta$-actin at 1:1000 dilution (Santa Cruz Biotechnology), mouse anti-human His-tag at 1:500 dilution (Merck, Germany). Detection was by horseradish peroxidase-conjugated secondary IgG-antibodies (Santa Cruz Biotechnology) as appropriate, and chemiluminescence reagent (Amersham/Pharmacia) followed by autoradiography (BioMaxfilm, Kodak).

In vivo tumor growth. Female BALB/C nude mice 4-6 weeks old were subcutaneously injected with breast cancer cells (Bcap37, $10^{7}$ cells suspended in $100 \mu \mathrm{l}$ PBS) into the right flank of each of the 48 mice. When the tumors had grown to about $90 \mathrm{~mm}^{3}$ in size, the mice were randomly divided into 6 treatment groups: dFdC with ZD55-dNKmut (Group 1) and Ad-dNKmut infected (Group 2), ZD55-dNKmut (Group 3) and Ad-dNKmut virus alone (Group 4), dFdC alone (Group 5), and PBS (control) group. All of the mice in the adenovirus treatment groups were intratumorally injected with a total dose of 109 pfu 3 times at $48-\mathrm{h}$ intervals and $\mathrm{dFdC}$ at $5 \mathrm{mg} / \mathrm{kg}$ i.p. twice on days 2-8 after virus injection. Tumor growth was monitored 5 days once and tumor volume $(\mathrm{V})$ was calculated using the formula: $\mathrm{V}\left(\mathrm{mm}^{3}\right)=1 / 2$ length $(\mathrm{mm}) \mathrm{x}$ width $(\mathrm{mm})^{2}$. Survival analysis expressed as time to progression was performed according to the method of Kaplan-Meier (logrank test for statistical significance). Tumor growth curves were compared using one-way ANOVA for significance.

Assessment of in vivo gene expression. The animals with tumor xenografts in the Bcap37 treatment groups (ZD55-dNKmut and ZD55 infected with $\mathrm{dFdC}, \mathrm{ZD} 55$ virus alone, and AddNKmut infected) were sacrificed on day 60. Tumors were excised and fixed in 10\% neutral formaldehyde for $6 \mathrm{~h}$, dehydrated with gradient ethanol, paraffin embedded, and sliced into $5 \mu \mathrm{m}$ sections for immunohistochemistry of E1A expression as described above using rabbit anti-Ad2/Ad5 E1A antibody. Animal experiments were performed in accordancy with the institutional ethics guidelines.

\section{Results}

Construction of recombinant adenovirus with Dm-dNKmut. Mutations of Dm-dNK at the last 10 amino acids in the sequence may increase the sensitivity of cells to $\mathrm{dFdC}$ and other purine nucleoside analogs as well as altering the expression location of Dm-dNK. The Dm-dNK mutants were created by site-directed mutagenesis in the amino acid sequence at sites of 244E, 245S, 251S, and 252R alternated to evaluate the enzyme activity (Fig. 1A). The viral E1B55-kDa gene was replaced by an expression cassette encoding dNKmut containing the sequence of CMV promoter + His-tag $(\mathrm{N})+$ multiple clone site + SV40 polyA into plasmid ZD55 and replication-deficient plasmid pShuttle (Ad) to generate plasmid ZD55-dNKmut and Ad-dNKmut (Fig. 1B). The infectivity of the cell lines were determined by the wild-type 5 adenovirus with expression of green fluorescence (Ad5-GFP), as shown in Fig. 1C. The cancer cell lines MGC-803 and SGC-7901 and the normal cell line MRC-5 exhibited nearly the same infectivity $(\sim 80-90 \%)$. 
A

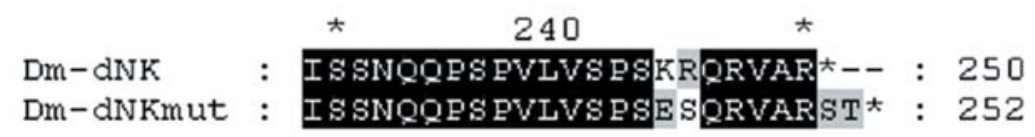

B
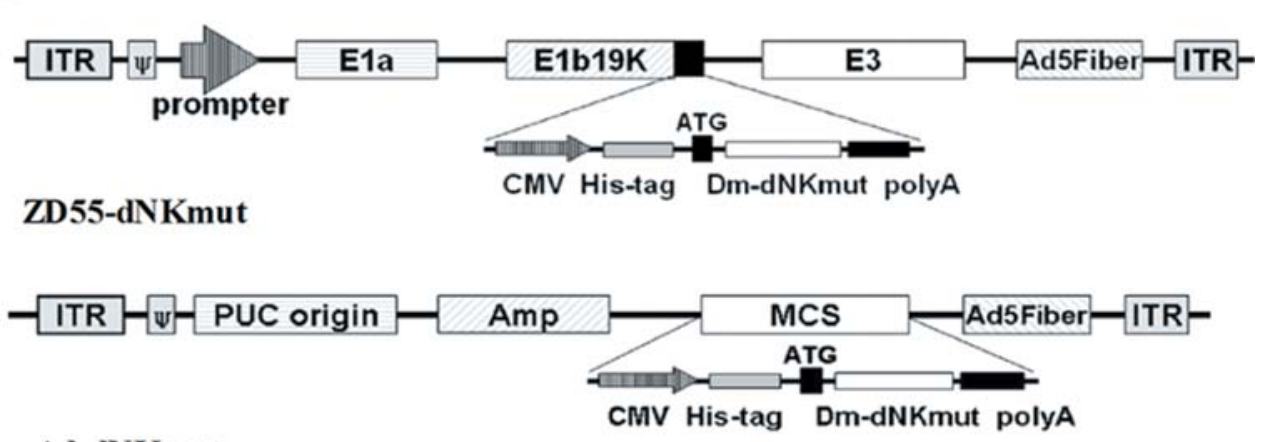

Ad-dNKmut

C

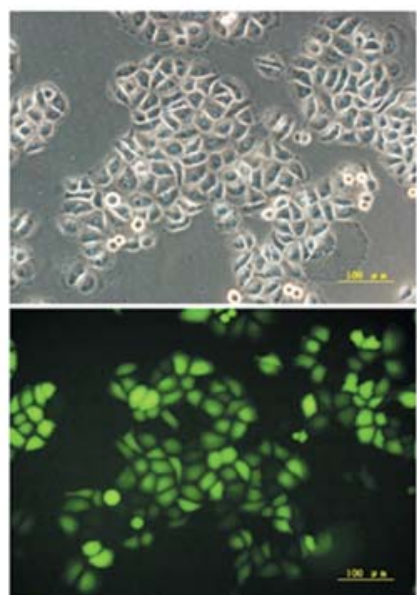

Bcap37

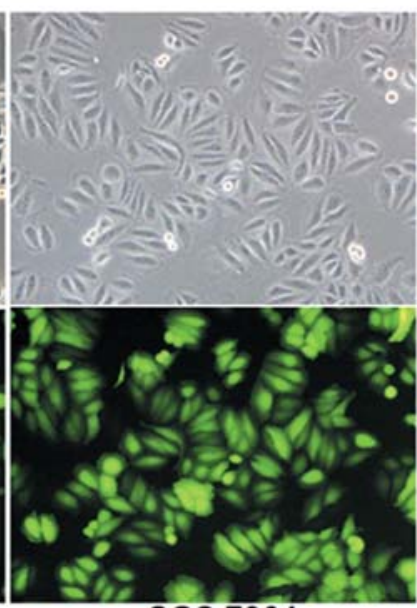

SGC-7901

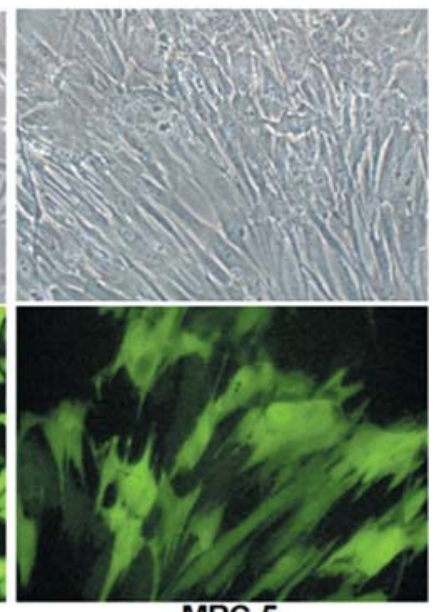

MRC-5

Figure 1. Construction and infectivity of ZD55-dNKmut and Ad-dNKmut. (A) Multiple alignment of the predicted amino acid sequences of Dm-dNK and Dm-dNKmut, but the last 10 amino acids were randomly alternated at site of 244E, 245S, 251S, and 252R. Amino acid sequences were obtained from the NCBI database. (B) To construct and infect ZD55-dNKmut, the E1B55-kDa gene was replaced by the expression cassette of Dm-dNKmut containing CMV promoter $+\operatorname{His}-\operatorname{tag}(\mathrm{N})+$ multiple clone site + SV40 polyA. The expression cassette was also inserted to the pShuttle vector to generate Ad-dNKmut (ITR, inverted terminal repeat; $\psi$, the adenovirus 5 packing signal; PUC origin, prokarotic origin of replication). (C) An overview of the infectivity in the 3 cell lines (MOI=100). More than $90 \%$ of the cells expressed GFP at MOI=100 with Ad5-GFP infected $48 \mathrm{~h}$ later.

Expression and enzyme activity of Dm-dNKmut. ZD55dNKmut expressed the Dm-dNKmut gene with a higher efficiency and activity in Bcap37 and SGC-7901 cancer cells compared with the replication-deficient adenovirus Ad-dNKmut. Our RT-PCR study results showed the selective expression of ZD55-dNKmut at MOI=10 after 2 days infection. The dNKmut gene of ZD55-dNKmut was somewhat higher than that of Ad-dNKmut in Bcap37 and SGC-7901 cancer cells; however, in MRC-5 cells, ZD55-dNKmut showed lower levels than that of Ad-dNKmut (Fig. 2A). Results from both the Western blot analysis (Fig. 2B) and immunoblot (Fig. 2C) of His-tag protein also demonstrated the same tendencies. His-tag TRITC also located the expression of Dm-dNKmut. Red fluorescence showed that the Dm-dNKmut was located in the cytosol and slightly in the nucleus. Enzyme activity was determined by the phosphorylation of dThd. The uninfected or infected with ZD55 vector adenovirus showed low levels of dThd phosphorylation, whereas the Bcap37 cancer cells infected with ZD55-dNKmut exhibited nearly 30-fold higher enzymatic activity than the parent cell line; 5 -fold higher than that of Ad-dNKmut, 1.5-fold higher than that of ZD55-dNK. In the normal cell lines MRC-5, activity of cells infected by ZD55-dNKmut was 10 -fold less than that in cancer cells (Fig. 2D).

Cell cytotoxic activity of Dm-dNKmut with $d F d C$. We investigated Dm-dNKmut for its potential use as a suicide gene in combination with the nucleoside analog $\mathrm{dFdC}$. As shown by the MTT assay results, the treatment of cells with ZD55dNKmut and Ad-dNKmut resulted in a dose-dependent reduction of cell viability within 5 days. With different concentrations of dFdC, ZD55-dNKmut and Ad-dNKmut were 
A

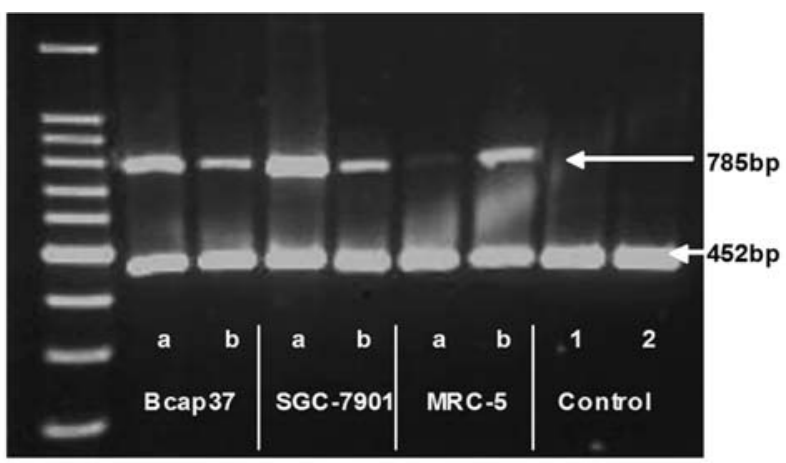

B

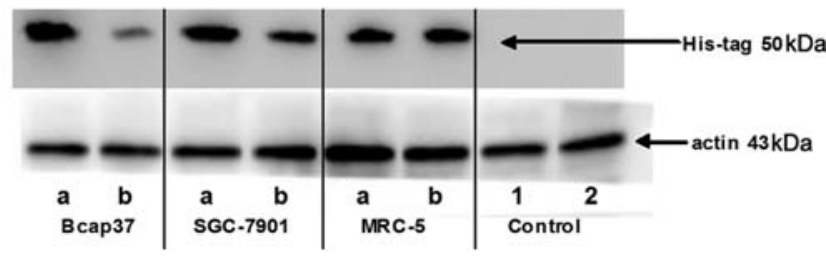

able to kill Bcap37 and SGC-7901 cancer cells more effectively than the ZD55 and Ad-blank. This occurred even at the very low concentration of $0.001 \mu \mathrm{M} \mathrm{dFdC}(\mathrm{p}<0.05)$. The nucleoside analog dose used was much lower than $\mathrm{IC}_{50}$. Cell killing rates of ZD55-dNKmut was slightly less than that of Ad-dNKmut in Bcap37 and not less than that of Ad-dNKmut in the SGC7901 cell line. The difference may be because of the different enzyme activity and sensitivity in various cell lines. However, in the normal MRC-5 cells, ZD55-dNKmut with drugs showed less cytotoxic than the other virus (Fig. 3A), with cell killing rates reaching a maximum of $50 \%$ lower than Ad-dNKmut, even when $\mathrm{dFdC}$ was added. The FACS assay also supported the results of MTT (Fig. 3B). The ZD55-dNKmut and Ad-dNKmut (MOI=10) with $1 \mu \mathrm{M}$ of $\mathrm{dFdC}$ induced the most cell apoptosis reaching as high as 76.9 and $76.04 \%$ (right upper and lower quadrant) in the Bcap37 cancer cells, which was $60 \%$ higher than the ZD55 with $\mathrm{dFdC}$. On the other hand, in the MRC-5 cells, with the presence of $1 \mu \mathrm{M}$ of $\mathrm{dFdC}, 7.24 \%$ of apoptosis was induced
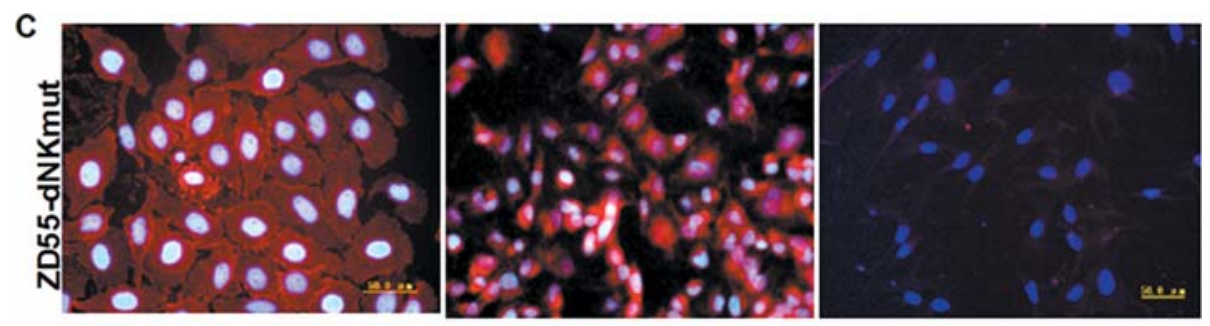

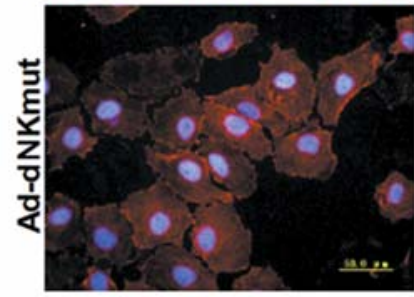

Bcap37

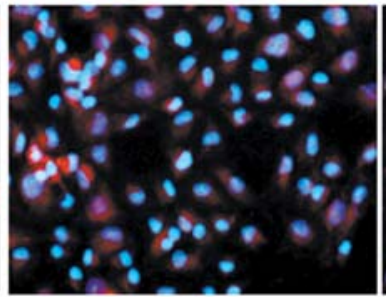

SGC-7901

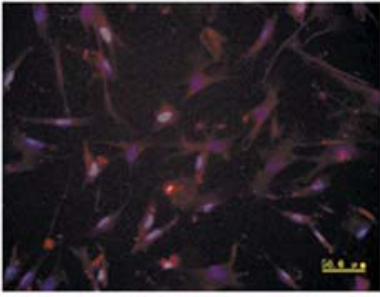

MRC-5

D

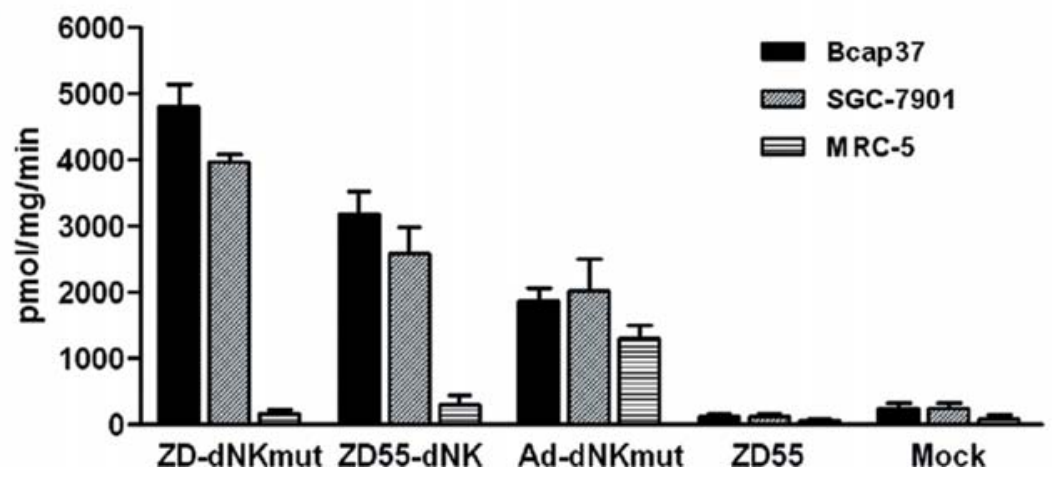

Figure 2. Expression and enzyme activity of Dm-dNKmut. Dm-dNKmut expression was quantified by (A) RT-polymerase chain reacton (PCR) analysis and (B) Western blot assay of His-tag expression in Bcap37, SGC-7901, and MRC-5 cells infected with (a) ZD55-dNKmut (b) Ad-dNKmut (1) ZD55 vector (2) Ad-blank at MOI=10 for $48 \mathrm{~h}$ after infection. The vector infected cells were used as control. The size of the amplified Dm-dNK and GAPDH was 785 and $452 \mathrm{bp}$, and 50 and $43 \mathrm{kDa}$ for the His-tag and actin, respectively. (C) We conducted immuno-fluorescence analysis to show His-tag expression 2 days after infection with the viruses of ZD55-dNKmut and Ad-dNKmut at MOI=10. We observed that the antibody of His-tag marked with TRITC showed that Dm-dNKmut was located in the cytosol, slightly in the nucleus. (D) The nucleoside kinase activity was quantified by the level of phosphorylation of dThd. The cells uninfected or infected with ZD55 vector adenovirus showed low levels of dThd phosphorylation, whereas the cancer cells infected with ZD55-dNKmut exhibited nearly 30-fold higher enzymatic activity than the parent cell line, 5-fold higher than that of Ad-dNKmut, and 1.5-fold higher than that of ZD55-dNK. The normal cell lines MRC-5 still showed stable low activity. 

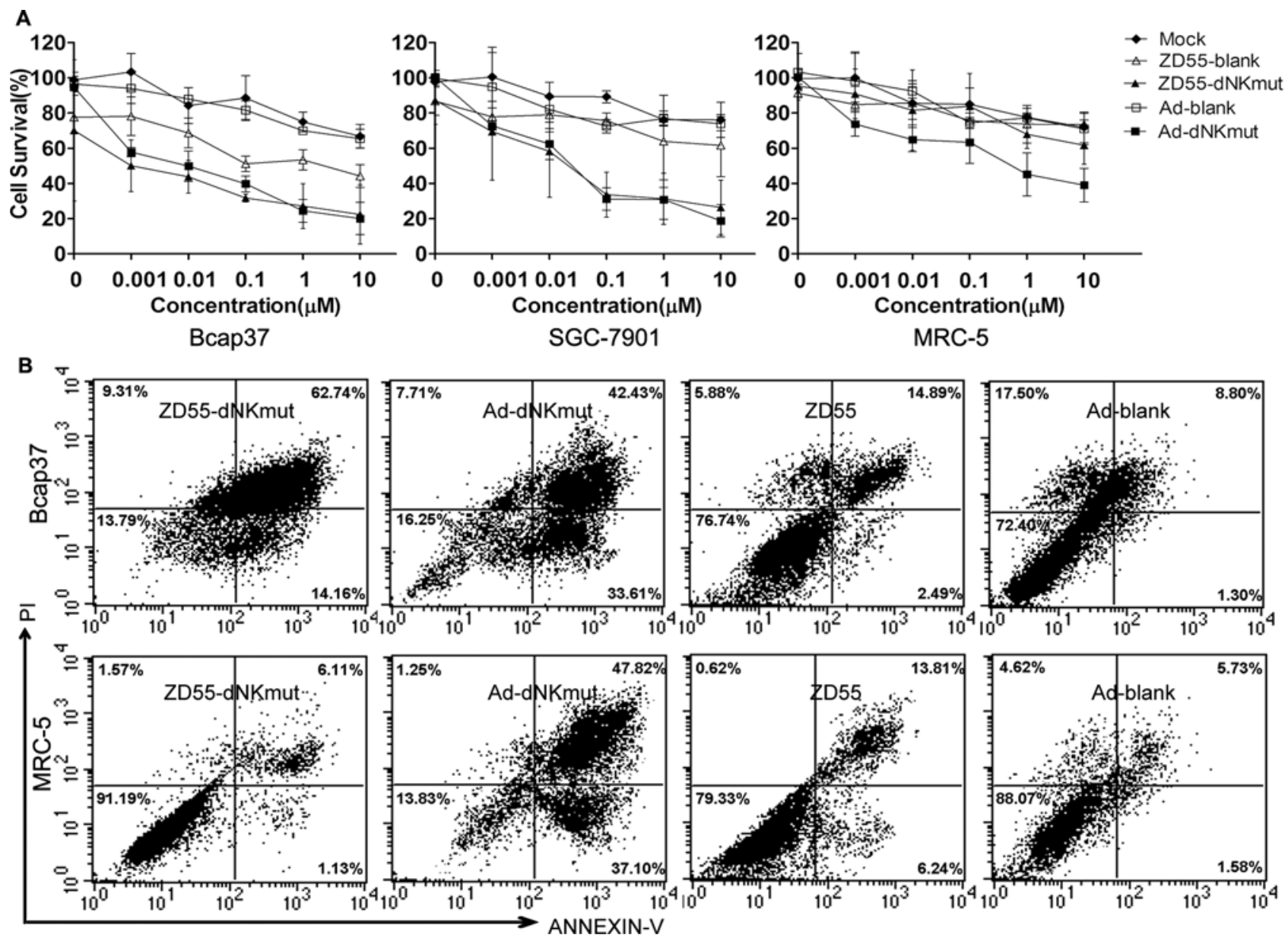

Figure 3. Selective cell killing activity of Dm-dNKmut with dFdC. (A) Cytotoxicity of Dm-dNKmut was assessed by MTT assay. Each cell line was infected with ZD55-dNKmut or Ad-dNK at an MOI=10 in combination with $\mathrm{dFdC}$ at does ranging from 0.001 to $10 \mu \mathrm{M}$. ZD55-dNKmut induced nearly equal proportions of cell death in cancer cells to that of Ad-dNKmut, but less profound cytotoxicity against normal cells. (B) The Bcap37 and MRC-5 cells were infected with ZD55-dNKmut, Ad-dNKmut, ZD55 and Ad-blank (MOI=10) for 2 days, and dFdC $(1 \mu \mathrm{M})$ added for 3 days. After the treatment, the percentage of apoptotic cells death was determined by FACS. ZD55-dNKmut induced the greatest selective apoptosis in cancer cells.

by ZD55-dNKmut, which was far less than ZD55 and AddNKmut. All of above data suggests that ZD55-dNKmut can sensitize cancer cell lines to $\mathrm{dFdC}$ without causing significant toxicity to normal cells.

Viral replication inhibited by Dm-dNKmut with $d F d C$. Surprisingly, in all of the cell lines, viral replication was detected at very low levels in the presence of $1 \mu \mathrm{mol} / 1$ of $\mathrm{dFdC}$ for the combination with ZD55-dNKmut, but not with ZD55 without Dm-dNK. Bcap37 cells supported higher levels of $10^{9} \mathrm{pfu} / \mathrm{ml}$ replication of the virus when ZD55 was used alone, but in the presence of $1 \mu \mathrm{M} \mathrm{dFdC}$, replication was detected at low levels of $<10^{6} \mathrm{pfu} / \mathrm{ml}$ for the ZD55-dNKmut (Fig. 4A). Despite a clear increase in replication over time without the drug, low doses of $\mathrm{dFdC}(1 \mu \mathrm{M})$ added $48 \mathrm{~h}$ after infection can lead to replicative inhibition of the virus for the combination ZD55-dNKmut. Attenuation was also observed to varying degrees in normal cell lines. The normal cells did not support replication of ZD55 vectors, but in combination with $1 \mu \mathrm{mol} / 1 \mathrm{dFdC}$, viral replication was potently attenuated. The E1A protein levels in Western blot experiments verify that $\mathrm{dFdC}$ activated by Dm-dNKmut could lead to inhibition of adenovirus replication. The E1A protein produced from the combination of ZD55-dNKmut with $\mathrm{dFdC}$ were at the lowest levels in both Bcap37 and MRC-5 cells (Fig. 4B). ZD55-dNKmut followed by the addition of $\mathrm{dFdC}$ attenuated E1A expression of adenovirus obviously in cancer cell lines, but no clear lane of E1A was detected in MRC-5 cell, except for wild-type Ad. These results show that viral E1A expression is not sufficient to overcome the $\mathrm{dFdC}$-induced arrest in response to DNA damage causing attenuation of replication. As expected, viral replication was decreased in the presence of this potent DNA-polymerase inhibitor, even at low concentrations.

Anti-tumor efficacy of Dm-dNKmut with dFdC in Bcap37 xenograft in vivo. The potency of the combination treatments was evaluated in an in vivo Bcap37 xenograft model with subcutaneous tumors treated with suboptimal doses of ZD55-dNKmut, Ad-dNKmut, and other control viruses with or without $\mathrm{dFdC}$ (Fig. 5). In animals treated with a virus dose at $1 \times 10^{9} \mathrm{pfu}$, median survival was 29 days for Ad-dNKmut virus and ZD55-dNKmut and 40 days with $\mathrm{dFdC}$ agent alone. However, when Ad-dNKmut and ZD55-dNKmut in combination with $5 \mathrm{mg} / \mathrm{kg}$ of $\mathrm{dFdC}$ was studied, time to progression was prolonged to 55 days and $>60$ days. Because the higher dose of virus $\left(1 \times 10^{9} \mathrm{pfu}\right)$ resulted in $>80 \%$ of animals not having progressed at the end of the study (60 days), no significant difference between ZD55-dNKmut and Ad-dNKmut 
A

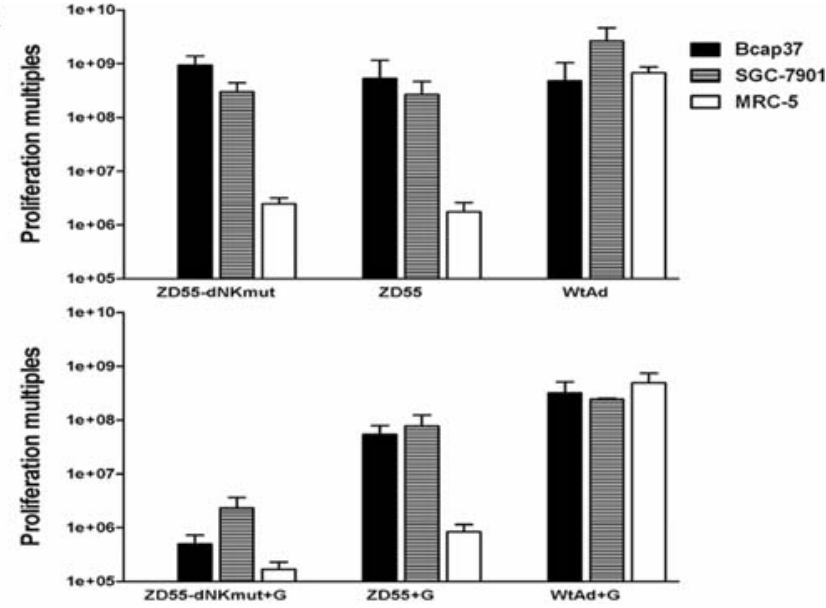

B
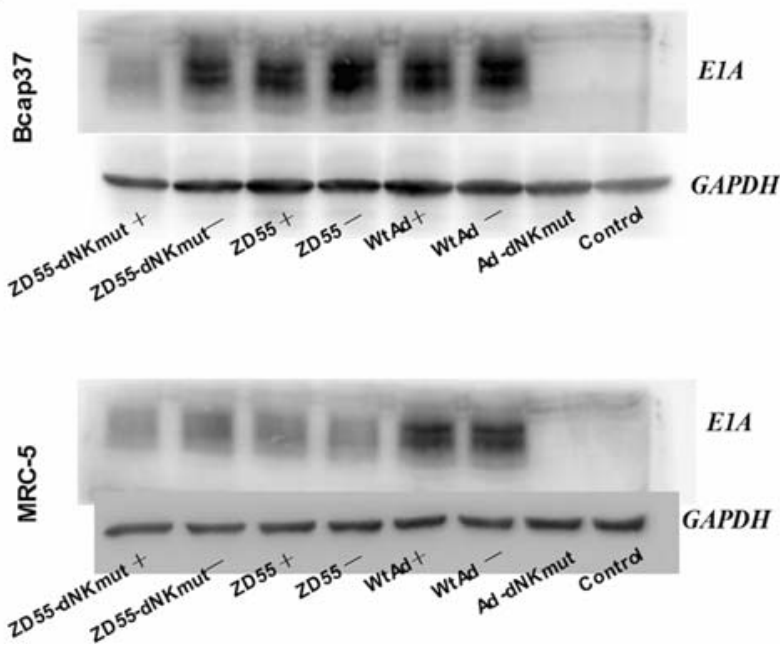

Figure 4. ZD55-dNKmut enhanced the inhibition effect of $\mathrm{dFdC}$ on viral replication in cancer cell lines Bcap37, SGC-7901, and in normal cell MRC-5. (A) Replication in cells was analyzed after 5 days of treatment with viruses alone $(\mathrm{MOI}=10)$ and in combination with $1 \mu \mathrm{M}$ of $\mathrm{dFdC}$. Drugs were added after virus infection (2 days). Both cells and media were analyzed by TCID50 assays. The titers of virus ZD55-dNKmut with $\mathrm{dFdC}$ (ZD55-dNKmut+G) were much lower ( $\leq 1000$-fold) than the level of viruses (ZD55, WtAd) alone or with $\mathrm{dFdC}$ in cell lines. (B) Western blot assay of E1A gene expression by ZD55-dNKmut, ZD55, WtAd with or without dFdC. With the therapy of ZD55-dNKmut combined with $\mathrm{dFdC}$, E1A of adenovirus was obviously attenuated in Bcap37 cells. However, in MRC-5 cells, ZD55-dNKmut and ZD55 did not produce clearly detectable E1A proteins, whether the drugs were added or not. Ad-dNKmut-infected cells and mock were used as a control.

with $\mathrm{dFdC}$ could be determined. Statistically significant anti-tumor efficacy in terms of tumor size was observed in ZD55-dNKmut and Ad-dNKmut with $\mathrm{dFdC}$-treated groups $\left(1076.26 \pm 623.21,964.86 \pm 369.33 \mathrm{~mm}^{3}\right)$ compared with the virus $\left(2123.43 \pm 442.72\right.$ and $\left.2865.66 \pm 332.49 \mathrm{~mm}^{3}\right)$ or drugs only $\left(2264.73 \pm 279.32 \mathrm{~mm}^{3}\right)$ and mock control group $\left(2766.76 \pm 431.54 \mathrm{~mm}^{3}\right)$, respectively, $\mathrm{p}<0.05$. The ZD55dNKmut-treated group appeared to be the most efficient in retarding tumor growth with the longer survival period.

Pathological examination. In a separate study, ZD55-dNKmut and ZD55 were combined with $5 \mathrm{mg} / \mathrm{kg}$ of $\mathrm{dFdC}$ and compared with the ZD55 adenovirus-alone infection. The result was a significant decrease of adenovirus-only in the treatment
$\mathbf{A}$
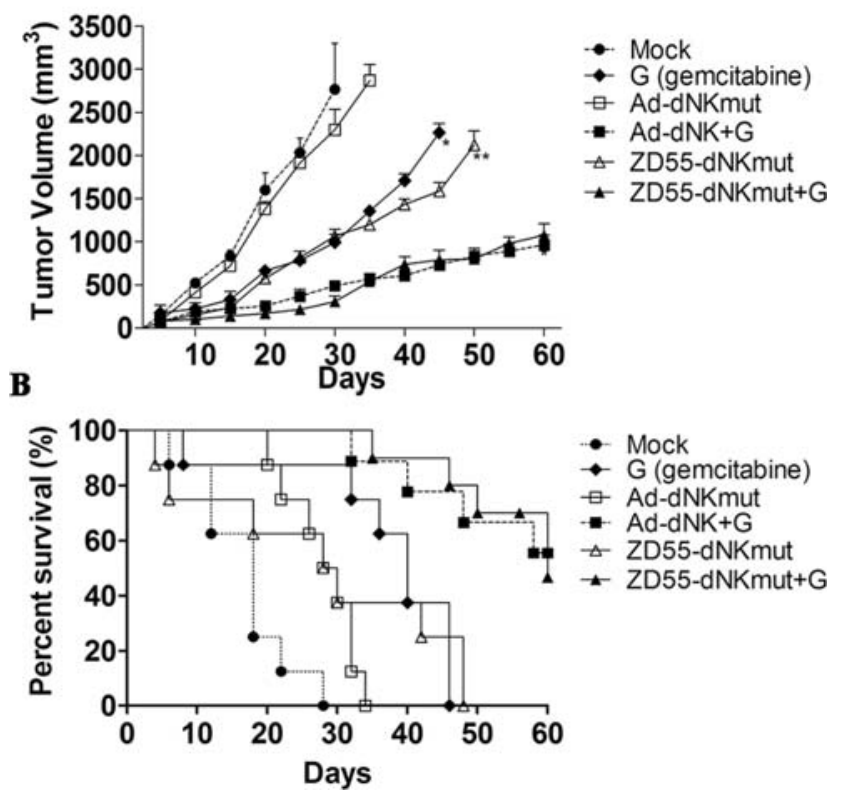

Figure 5. In vivo anti-tumor potential of ZD55-dNKmut and Ad-dNKmut in breast cancer Bcap37 xenograft mouse model. Xenografted mice ( 8 in each group) were given intratumor injections of the indicated viruses on 5 alternate days with a total dose of $10^{9} \mathrm{pfu} / \mathrm{mouse}$. (A) Tumor volume $\left(\mathrm{mm}^{3}\right)$ was monitored up until day 60 (the day they had to be sacrificed) and up until day 29 for the PBS-treated control (mock) group. ${ }^{* *} \mathrm{P}<0.01$ and ${ }^{*} \mathrm{P}<0.05$ for combination treatments compared with either single-agent treatment and ${ }^{*} \mathrm{P}<0.05$ for each treatment compared with mock-treated animals. Significance was determined by one-way ANOVA analysis. (B) Kaplan-Meier curves for the indicated treatment groups. All combinations were significantly different from single-agent treatments with ${ }^{*} \mathrm{P}<0.05$.
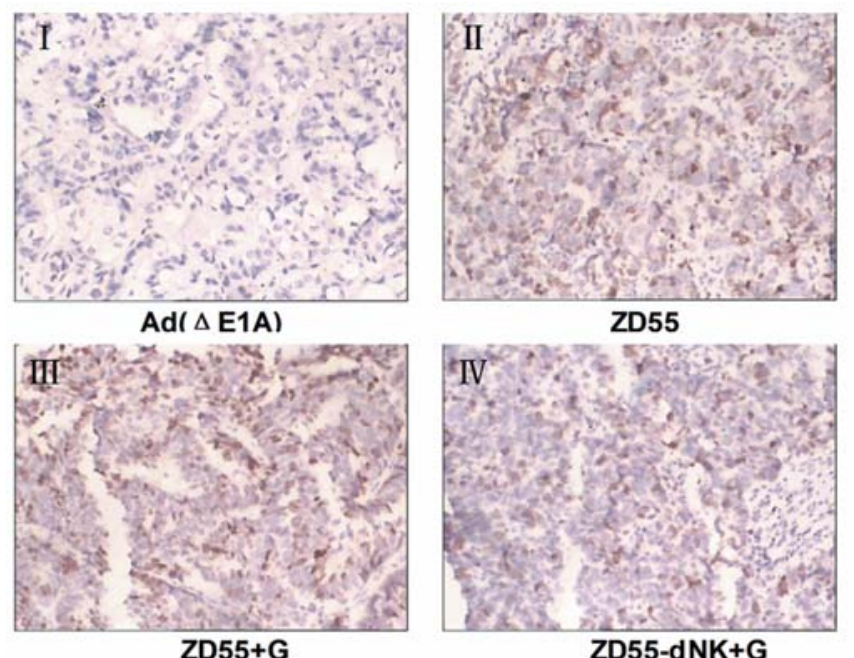

Figure 6. Immunohistochemistry analysis of E1A protein in vivo. Expression of E1A was demonstrated by immunohistochemical staining in Bcap37 xenograft mice treated with (I) Ad-dNKmut, (II) ZD55 virus alone, (III) ZD55 infected with $\mathrm{dFdC}$ and (IV) ZD55-dNKmut infected with $\mathrm{dFdC}$, four groups of tumor slice were evaluated from each treatment group, which presented as concentrated positive staining of nucleus. Positive, brown (x100).

group receiving ZD55-dNKmut combined with drug. The corresponding tumor samples stained positive for E1A expression up to 60 days after viral administration both with and without $\mathrm{dFdC}$ (Fig. 6). E1A-positive cells were 
detected in xenografts treated with the combinations up to 50 days after treatment. E1-deleted, non-replicating adenovirus Ad series had no efficacy in this model (Fig. 6I). Expression of E1A was demonstrated by immunohistochemical staining in Bcap37 xenograft mice treated with ZD55-dNKmut or ZD55, which presented as concentrated positive staining of nucleus. The expression level of E1A in cells treated with ZD55-dNKmut+dFdC was comparable lower than those treated with ZD55+dFdC (Fig. 6III and IV). The results indicate that an inhibitory effect the on replication of adenovirus comes from the recombiant Dm-dNKmut oncolytic adenovirus in xenografts in vivo.

\section{Discussion}

Cancer cells are genetically and phenotypically complex and frequently harbor multiple abnormalities (21). Using monotherapies with single agents or focusing on single oncogene therapy it is difficult to achieve the desired therapeutic effect for malignancies, the perspective of a mechanism of simple destruction, such as suicide-gene/chemotherapy strategy is found to be more feasible from complex tumorigenic genes (22).

The most widely used suicide-gene/chemotherapy system is HSV-tk/GCV (herpes viruses carrying a TK that can phosphorylate dCyd) as well as TMP and dCMP (23). The herpetic kinases with the relatively broad substrate specificity have many features in common with the mammalian TK2, $\mathrm{dCK}$, and dGK. However, the isolated deoxyribonucleoside kinase from Drosophila melanogaster Dm-dNK appears to possess the ability to phosphorylate all four deoxyribonucleosides with higher catalytic activity for pyrimidine than that of TK (24). Recently, a cDNA from Dm-dNK was found to be homologous to the cDNA of mammalian TK2, dCK, and $\mathrm{dGK}$, except for a unique C-terminal amino acid sequence, which appeared to be important for the catalytic activity and also contained a nuclear localization signal, especially in the last 10 amino acids (5). We randomly alternated the last 10 amino acids to create dNKmut (244E, 245S, 251S, 252R) to express both in the nucleus and cytosol (Fig. 1C). The nucleoside kinase activity proved to be higher in vitro than wild-type Dm-dNK (Fig. 2D). Since the nucleoside analogs may freely traverse the nuclear envelope, phosphorylating in either of the two subcellular compartments (25). The recombinant dNKmut was more favorable for medical therapy.

We constructed a replicative oncolytic adenovirus carrying dNKmut (ZD55-dNKmut). For the first time, we used DmdNK mutants to combine an oncolytic effect with the nucleoside analog substrate $\mathrm{dFdC}$, which has higher affinity in vitro to the enzyme compared with other purine deoxyribonucleosides (26). Our data suggested that less toxic doses of $\mathrm{dFdC}$ could efficiently target breast and gastric adenocarcinomas when combined with dNKmut and induce apoptosis in high proportion of cancer cells. The normal MRC-5 cells were barely influenced at all (Fig. 3B).

To evaluate the oncolytic effects of ZD55-dNKmut, we designed our study with the replication-deficient adenovirus as our control. Previous studies demonstrated the application of CRAd vector always possesses a stronger and higher effect than the RDAd vector in gene therapy with an oncolytic effect (15-18). ZD55-dNKmut was supposed to express enzymatical activity of dNKmut increased with the adenovirus genome amplification. Interestingly, there was no obviously potent increase in cell death in response to the combined treatment depended on virus, even attenuation of replication with ZD55-dNKmut in gastric cancer cells combined with $1 \mu \mathrm{M}$ of dFdC was observed 5 days post-infection (Fig. 4A). We considered that the $\mathrm{dFdC}$ conversion to its triphosphate form (TP) phosphorylated by dNKmut may lead to more apoptosis of the cancer cells and adenovirus, as shown in our Western blotting of adenovirus E1A protein and titer assays (Fig. 4B). The replication of adenovirus could be controlled, to some extent, by certain types of dNKmut that possess nucleoside analogs to protect the normal cells, otherwise, $\mathrm{dFdC}$ itself also could block replication of viruses due to $\mathrm{dFdC}$-induced delay in G1/S-cell cycle progression, with repression of cyclin $\mathrm{E}$ and cdc25A $(27,28)$. In more recent reports by Morris using wild-type replicating adenoviruses containing an HSV-TK transgene, no augmentation of killing was observed after the addition of GCV (29). The controversial conclusion could be explained that GCV-TP inhibited the virus vector expression of HSV-TK continuously through inducing the death of virus, as is our finding.

Although Ad-dNKmut combined with $\mathrm{dFdC}$ showed no less killing activity than ZD55-dNKmut in the breast carcinoma cell line Bcap37 or a tumor xenograft mouse model (Fig. 5A), the activated $\mathrm{dFdC}$ played an important role in inhibiting viral-replication. Activated $\mathrm{dFdC}$ actually is limited by low selectivity toward the target cells and low transduction efficiency. Futhermore, severe side effects resulting from excessive replication were the key obstacles of CRCAds in the clinical treatment of cancer gene therapy. Our in vitro data and pathological examinations demonstrated that expression of adenovirus early transcription gene E1A could be controlled (Fig. 6), to some extent, and was associated with apoptosis in the majority of ZD55-dNKmut/dFdC-treated tumor cells, even when the adenovirus vectors ZD55 amplification in the normal cells expressed suicide gene to produce unwanted effects. So our scenario may be a potentially novel, efficient, and selective anti-cancerous agent with considerably low side effects as compared with CRAd.

The broad substrate specificity and high catalytic efficiency compared with other kinases makes the Dm-dNK family (especially Dm-dNKmut, with its even higher catalytic rates for deoxyribonucleosides) the attractive feature in our strategy of dFdC combined with dNKmut. dFdC with less toxic doses under dNKmut catalysis enhanced cytotoxicity and had a synergistic effect of suppressing excessive adenovirus replication. Combining the dNKmut therapy with other antimetabolites to achieve synergistic effects may be another approach to enhance the efficiency of nucleoside kinase suicide gene therapy, without breaking the chemical activity of the drug itself. The dNKmut enzyme also makes it possible to achieve various functions with one or one more substrates simultaneously.

\section{Acknowledgements}

This work was supported by grants from the National Natural Science Foundation of China (No. 81071900) and Hi-Tech 
Research Development Program of China (863 Program, 2006AA02Z493).

\section{References}

1. Zheng X, Johansson M and Karlsson A: Retroviral transduction of cancer cell lines with the gene encoding Drosophila melanogaster multisubstrate deoxyribonucleoside kinase. J Biol Chem 275: 39125-39129, 2000

2. Zheng X, Johansson $M$ and Karlsson A: Bystander effects of cancer cell lines transduced with the multisubstrate deoxyribonucleoside kinase of Drosophila melanogaster and synergistic enhancement by hydroxyurea. Mol Pharmacol 60: 262-266, 2001.

3. Zheng X, Johansson $\mathrm{M}$ and Karlsson A: Nucleoside analog cytotoxicity and bystander cell killing of cancer cells expressing Drosophila melanogaster deoxyribonucleoside kinase in the nucleus or cytosol. Biochem Biophys Res Commun 289: 229-233, 2001.

4. Betham B, Shalhout S, Marquez VE and Bhagwat AS: Use of Drosophila deoxynucleoside kinase to study mechanism of toxicity and mutagenicity of deoxycytidine analogs in Escherichia coli. DNA Repair 9: 153-160, 2010.

5. Munch-Petersen B, Knecht W, Lenz C, Sondergaard L and Piskur J: Functional expression of a multisubstrate deoxyribonucleoside kinase from Drosophila melanogaster and its Cterminal deletion mutants. J Biol Chem 275: 6673-6679, 2000.

6. Solaroli N, Bjerke M, Amiri MH, Johansson M and Karlsson A: Active site mutants of Drosophila melanogaster multisubstrate deoxyribonucleoside kinase. Eur J Biochem 270: 2879-2884, 2003.

7. Solaroli N, Johansson M, Balzarini J and Karlsson A: Enhanced toxicity of purine nucleoside analogs in cells expressing Drosophila melanogaster nucleoside kinase mutants. Gene Ther 14: 86-92, 2007.

8. Mikkelsen NE, Munch-Petersen B and Eklund H: Structural studies of nucleoside analog and feedback inhibitor binding to Drosophila melanogaster multisubstrate deoxyribonucleoside kinase. FEBS J 275: 2151-2160, 2008.

9. Chen MS and Prusoff WH: Association of thymidylate kinase activity with pyrimidine deoxyribonucleoside kinase induced by herpes simplex virus. J Biol Chem 253: 1325-1327, 1978.

10. Beltinger C, Fulda S, Kammertoens T, Meyer E, Uckert W and Debatin KM: Herpes simplex virus thymidine kinase/ganciclovirinduced apoptosis involves ligand-independent death receptor aggregation and activation of caspases. Proc Natl Acad Sci USA 96: 8699-8704, 1999.

11. Wildner $\mathrm{O}$ and Morris JC: The role of the E1B $55 \mathrm{kDa}$ gene product in oncolytic adenoviral vectors expressing herpes simplex virus-tk: assessment of antitumor efficacy and toxicity. Cancer Res 60: 4167-4174, 2000

12. Raki M, Hakkarainen T, Bauerschmitz GJ, et al: Utility of $\mathrm{TK} / \mathrm{GCV}$ in the context of highly effective oncolysis mediated by a serotype 3 receptor targeted oncolytic adenovirus. Gene Ther 14: 1380-1388, 2007.

13. Lambright ES, Amin K, Wiewrodt R, et al: Inclusion of the herpes simplex thymidine kinase gene in a replicating adenovirus does not augment antitumor efficacy. Gene Ther 8: 946-953, 2001.
14. Zhang ZL, Zou WG, Luo CX, et al: An armed oncolytic adenovirus system, ZD55-gene, demonstrating potent antitumoral efficacy. Cell Res 13: 481-489, 2003.

15. Wang ZG, Zhao W, Ramachandra M and Seth P: An oncolytic adenovirus expressing soluble transforming growth factor-beta type II receptor for targeting breast cancer: in vitro evaluation. Mol Cancer Ther 5: 367-373, 2006.

16. Ranki T, Sarkioja M, Hakkarainen T, von Smitten K, Kanerva A and Hemminki A: Systemic efficacy of oncolytic adenoviruses in imagable orthotopic models of hormone refractory metastatic breast cancer. Int J Cancer 121: 165-174, 2007.

17. Gan Y, Gu J, Cai X, Hu J, Liu XY and Zhao X: Adenovirusmediated HCCS1 overexpression elicits a potent antitumor efficacy on human colorectal cancer and hepatoma cells both in vitro and in vivo. Cancer Gene Ther 15: 808-816, 2008.

18. Chen L, Chen D, Gong M, et al: Concomitant use of Ad5/35 chimeric oncolytic adenovirus with TRAIL gene and taxol produces synergistic cytotoxicity in gastric cancer cells. Cancer Lett 284: 141-148, 2009.

19. Reid T, Galanis E, Abbruzzese J, et al: Hepatic arterial infusion of a replication-selective oncolytic adenovirus (d11520): phase II viral, immunologic, and clinical endpoints. Cancer Res 62: 6070-6079, 2002

20. Sandrini MP, Clausen AR, On SL, Aarestrup FM, MunchPetersen B and Piskur J: Nucleoside analogues are activated by bacterial deoxyribonucleoside kinases in a species-specific manner. J Antimicrob Chemother 60: 510-520, 2007.

21. El-Serag HB and Rudolph KL: Hepatocellular carcinoma: epidemiology and molecular carcinogenesis. Gastroenterology 132: 2557-2576, 2007

22. Wierdl M and Potter PM: Update on gene therapy approaches for cancer. Curr Hematol Rep 4: 294-299, 2005.

23. Fillat C, Carrio M, Cascante A and Sangro B: Suicide gene therapy mediated by the Herpes Simplex virus thymidine kinase gene/Ganciclovir system: fifteen years of application. Curr Gene Ther 3: 13-26, 2003.

24. Johansson M, van Rompay AR, Degreve B, Balzarini J and Karlsson A: Cloning and characterization of the multisubstrate deoxyribonucleoside kinase of Drosophila melanogaster. J Biol Chem 274: 23814-23819, 1999.

25. Johansson M, Brismar S and Karlsson A: Human deoxycytidine kinase is located in the cell nucleus. Proc Natl Acad Sci USA 94: 11941-11945, 1997.

26. Knecht W, Mikkelsen NE, Clausen AR, et al: Drosophila melanogaster deoxyribonucleoside kinase activates gemcitabine. Biochem Biophys Res Commun 382: 430-433, 2009.

27. Jordheim LP, Galmarini CM, Dumontet C: Gemcitabine resistance due to deoxycytidine kinase deficiency can be reverted by fruitfly deoxynucleoside kinase, DmdNK, in human uterine sarcoma cells. Cancer Chemother Pharmacol 58: $547-$ 554, 2006.

28. Cappella P, Tomasoni D, Faretta M, et al: Cell cycle effects of gemcitabine. Int J Cancer 93: 401-408, 2001.

29. Morris JC, Wildner O: Therapy of head and neck squamous cell carcinoma with an oncolytic adenovirus expressing HSV-tk. Mol Ther 1: 56-62, 2000. 